\title{
A Test Environment for Power Semiconductor Devices Using a Gate-Boosting Circuit
}

\author{
Martin Hochberg, Associate Member, IEEE, Martin Sack, Member, IEEE, Georg Mueller, Member, IEEE,
}

\begin{abstract}
For semiconductor-based pulsed power generators, short turn-on and turn-off times of the employed switching elements are especially in hard switching condition of advantage. In order to enhance switching speeds for standard devices under hard switching conditions, we propose a capacitively coupled gate-boosting circuit with gate drive voltages of up to $80 \mathrm{~V}$. A low inductance test circuit has been set up to evaluate different switching elements under varying driving conditions. Beside single pulse operation, the setup allows for burst mode and continuous repetitive switching. The flexible design of the setup allows for testing MOSFETs as well as IGBTs. Exemplary, we show results for a $\mathrm{SiC}$ MOSFET and a standard IGBT. By applying $80 \mathrm{~V}$ gate drive voltage, the current rise rate of the $\mathrm{SiC}$ MOSFET could be increased by a factor of 3.5 up to $2.6 \mathrm{kA} / \mu \mathrm{s}$. For standard IGBT devices we achieved an eight-fold increase in current rise rate to up to $4.2 \mathrm{kA} / \mu \mathrm{s}$. We verified a reproducible pulse shape for over $10^{8}$ pulses. Additionally, the gate drive circuit is able to drive the device in $3 \mathrm{MHz}$ burst mode at $250 \mathrm{~A}$ peak collector current.
\end{abstract}

Index Terms-Pulse power systems, Pulse generation, Pulse circuits, Pulse power system switches, Power semiconductor devices, Insulated gate bipolar transistors, Power MOSFETs

\section{INTRODUCTION}

W HEN designing semiconductor-based pulsed power generators, the choice of the switching element is important. Whereas silicon metal-oxide-semiconductor fieldeffect transistors (MOSFETs) are known for their fast switching speed [1], they suffer from increased on-state resistance with increased blocking voltage [2]. Using wide-bandgap semiconductors such as $\mathrm{SiC}$, the on-state resistance can be lowered [3]. As economic alternative for reduced conduction losses, insulated-gate bipolar transistors (IGBTs) may be used [2]. Although their switching speed is lower when compared to MOSFETs, advanced gate drive circuits may reduce switching times [4].

Since datasheets contain experimental results for well defined test circuits only, evaluation tests for different devices specifically tailored to the intended application become necessary prior to circuit design. Especially when operating the devices near or beyond the specified limits, long term experiments can be used to detect slow device degradation [5] and hence to ensure reliable operation of the final generator. In this paper we present a gate drive circuit being able to speed up switching of both MOSFET and IGBT devices. In order to study the switching behavior of the devices under test, we

The authors are with Karlsruhe Institute of Technology, Institute for Pulsed Power and Microwave Technology, Eggenstein-Leopoldshafen 76344, Germany (e-mail: martin.hochberg@kit.edu; martin.sack@kit.edu; georg.mueller@kit.edu). Manuscript submitted November 30, 2015; accepted May 9, 2016. Digital Object Identifier 10.1109/TPS.2016.2586518 set up a low inductivity pulse circuit for studies in single pulse, burst and repetitive switching mode. With the obtained information, more precise predictions of the final generator behavior become feasible.

\section{EXPERIMENTAL SETUP}

\section{A. Gate drive circuit}

To achieve fast switching speeds, fast charging of the gate capacitance is important for both MOSFETs and IGBTs. Parasitic inductances in the gate drive circuit cause a time delay for turn-on and turn-off [6]. Additionally, they necessitate a damping resistor ("gate resistor") in series to the gate capacitance to prevent oscillations. The standard gate drive circuit consists of a push-pull stage charging the gate via a gate resistor with a voltage equal to the final gate voltage (for standard IGBTs $+15 \mathrm{~V}$ to $-15 \mathrm{~V}$ ) [2]. The combination of gate resistor, gate capacitance and parasitic inductance, however, forms a RLC low-pass causing a low rise rate of the gate voltage. One approach for faster gate charging is the so called "gate boosting" [2]. A higher voltage than the final gate voltage is applied to the gate during the charging process for achieving higher gate charging currents. Some authors [2] use two different driving voltages during turn-on and steady-on (e.g. $+24 \mathrm{~V}$ and $+15 \mathrm{~V}$ ), whereas others propose to discharge a capacitor (charged at $+60 \mathrm{~V}$ ) into the gate and clamp the gate voltage to less than $15 \mathrm{~V}$ using a TransientVoltage-Suppression Diode [7]. These approaches work well, however, the circuit topology is complex and uses additional active elements as compared to a standard push-pull gate drive. The gate drive circuit under investigation in this paper is shown in fig. 1. Therein, the switching element $\mathrm{T} 1$ is considered to be an ideal semiconductor switch, whereas its internal components such as gate capacitance $\mathrm{C} 2$, parasitic device inductance L1 are drawn as discrete components. The outline of the device package with the external device leads (collector, gate and emitter) is indicated by dashed lines. The gate drive consists of a fast push-pull MOSFET stage (M1, M2; Fairchild FDT86102LZ [8]) receiving the gate signal. V1 represents the gate drive voltage and can be adjusted for experiments between $15 \mathrm{~V}$ and $80 \mathrm{~V}$. Once M1 switches on, the gate drive voltage is divided by the damped capacitive divider $\mathrm{C} 1$ and the gate capacitance $\mathrm{C} 2$. The damping resistors $\mathrm{R} 1, \mathrm{R} 2$ and $\mathrm{R} 3$ are used to dampen oscillations that may arise from the combination of the before-mentioned capacitors and parasitic inductances. To compensate for charge loss during long pulses, additional charge is supplied via R5, whereas a maximum gate voltage of $15 \mathrm{~V}$ in steady on-state is ensured by the combination of 
D1 and R4. During turn-off, the capacitive divider enables fast discharging of the gate capacitance. In steady-off state, diode D2 prevents unintended gate charging. Therefore, a low resistance path to ground can be created by choosing R2 smaller in comparison to R1. As R1 and R2 also dampen oscillations between the output capacitances of M1 and M2, their lower limit is determined by the parasitic inductances in the layout.

Depending on the switching element under test, the coupling capacitor $\mathrm{C} 1$ which determines the final gate voltage $\mathrm{V}_{G}$ has to be adjusted to the driving voltage $\mathrm{V} 1$ and the respective gate capacitance $\mathrm{C} 2$ of the device. The final gate voltage $\mathrm{V}_{G}$ can be calculated according to eq. 1 :

$$
V_{G}=\frac{V_{1} \cdot C_{1}}{C_{1}+C_{2}}
$$

The coupling capacitor may be adjusted to reach a gateemitter voltage slightly above the recommended value in the datasheet without any collector-emitter voltage V2 applied. Thereby, the additional charge required for charging the gate-collector capacitance with rising collector-emitter voltage ("Miller-capacitance") is accounted for. When choosing C1, the gate charge as given in the datasheet must not be exceeded. Otherwise, transient overvoltage may damage the device's gate structure. In the PCB layout of the circuit, special care has been taken with respect to low inductance connections in the gate path - allowing for the smallest damping resistors possible. In addition, a sufficient distance is to be kept from the gate connection to adjacent planes in order to minimize stray capacitances.

Due to the ease of adaptation to different switching elements and the adjustability of the driving voltage, this simple gate drive circuit allows us to study the influence of fast gate charging on different switching elements. To demonstrate the effect of a capacitively coupled gate drive, we chose a standard IGBT in a TO-247 housing with high pulse current handling capability (maximum rated pulse current $320 \mathrm{~A}$ NGTB40N120IHLWG, ON Semiconductor [9]). As emerging technology [3], the second device under test was a fast $\mathrm{SiC}$ MOSFET with low conduction losses (C2M0080120D, Cree Inc. [10]) in the same package.

\section{B. Pulse circuit}

In some datasheets, values for current rise and fall times are given for an inductive load. We found that the measured switching times in hard switching conditions with a mainly ohmic load vary significantly from the given values. As for fast pulse generators low inductance setup is important, our test environment was designed for hard switching conditions. The setup of the pulse circuit is shown in fig. 2. Capacitor C3 (consisting of several pulse capacitors in parallel) is charged to voltage V2 (up to $1 \mathrm{kV}$ ) via diode D3. Subsequently, switching element T1 is switched on and discharges the capacitor over load resistor R6 and shunt resistor R7. Hence, the measured current pulse is negative with respect to ground. For single pulse experiments, the load resistor consists of several ceramic pulse resistors connected in parallel. In case of repetitive

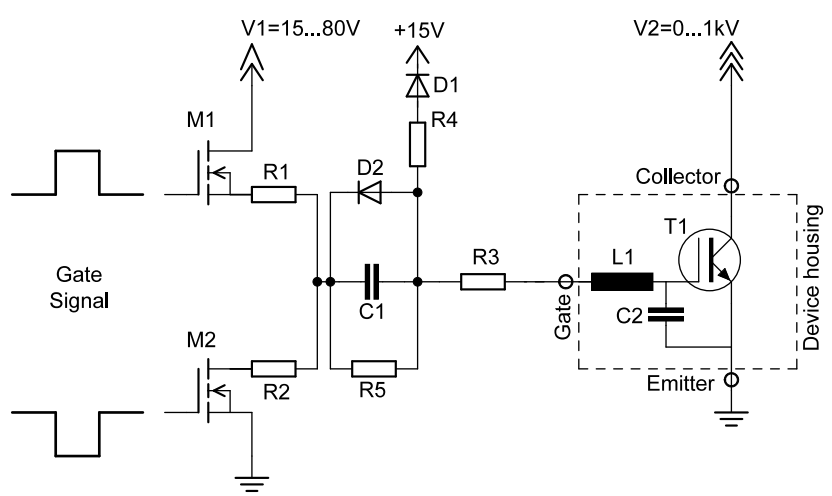

Fig. 1: The gate drive circuit under investigation. Here, the IGBT T1 is considered an ideal semiconductor switch, its internal components such as internal inductance L1 and gate capacitance C2 are shown as discrete components. The physical device housing with the collector, gate and emitter pins is indicated by dashed lines. On turn-on, the MOSFET halfbridge switches the gate drive voltage onto the capacitive divider comprising the coupling capacitor $\mathrm{C} 1$ and the gate capacitance $\mathrm{C} 2$.

experiments where power dissipation in the load is high, a coaxial water resistor has been used. For cooling, the switching element has been equipped with a water-cooled heatsink whereas the water resistor was immersed in cooling water. The used shunt resistor consists of $301 \Omega$ SMD resistors

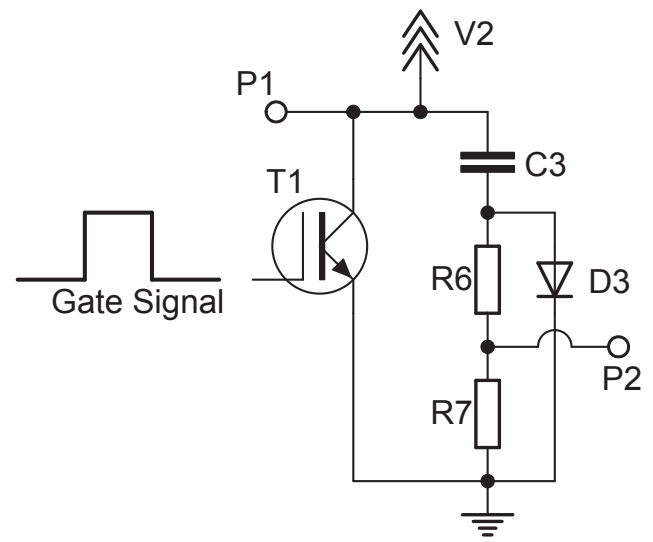

Fig. 2: Schematic of the designed pulse circuit. It is composed of the pulse capacitor $\mathrm{C} 3$ being charged to voltage V2 via diode D3. When switching element T1 is switched on, the capacitor discharges over load resistor R6 and shunt resistor $\mathrm{R} 7$, resulting in a negative current peak with respect to ground. The collector-emitter voltage is measured at point P1, the current signal is measured at $\mathrm{P} 2$, both with respect to ground.

capable of pulsed overload connected in parallel in a coaxial configuration similar to a shunt resistor presented earlier [11]. Its frequency response is flat up to the measurement limit of $1 \mathrm{GHz}$. For the experiments with high repetition rate, the shunt resistor was replaced by a current monitor (Pearson type 110). All data was recorded using an oscilloscope with $2 \mathrm{GHz}$ bandwidth (LeCroy WaveRunner 204MXi-A). Voltage has been decoupled by means of high voltage probes with a 
rise time of $1.2 \mathrm{~ns}$ (Phillips PM8932). The cable propagation time difference between current and voltage measurement was measured to be $2 \mathrm{~ns}$ and hence negligible for the presented data. The measured inductance of the pulse circuit (excluding the switching element) was measured by means of a LCR bridge (Hameg HM8118) to be below $60 \mathrm{nH}$ when using the ceramic resistors and below $80 \mathrm{nH}$ when using the water resistor. For the load to be considered ohmic, the rise time of the switching element must be significantly larger than the maximum current rise time $\tau$ given as ratio of the circuit's inductance L over the circuit's resistance R (see eq. 2).

$$
\tau=\frac{L}{R}
$$

Fig. 3 shows collector current and collector-emitter voltage curves for a pulse with a rise and fall time in the order of $50 \mathrm{~ns}$ for a resistance of $3.75 \Omega$ in the $60 \mathrm{nH}$ circuit (resulting maximum current rise time: $21 \mathrm{~ns}$ ). As can be seen, both curves are identical and no significant phase lag between current and voltage is observable. Hence, the designed pulse circuit is suitable for experiments under hard switching conditions, if $\tau$ is kept sufficiently small.

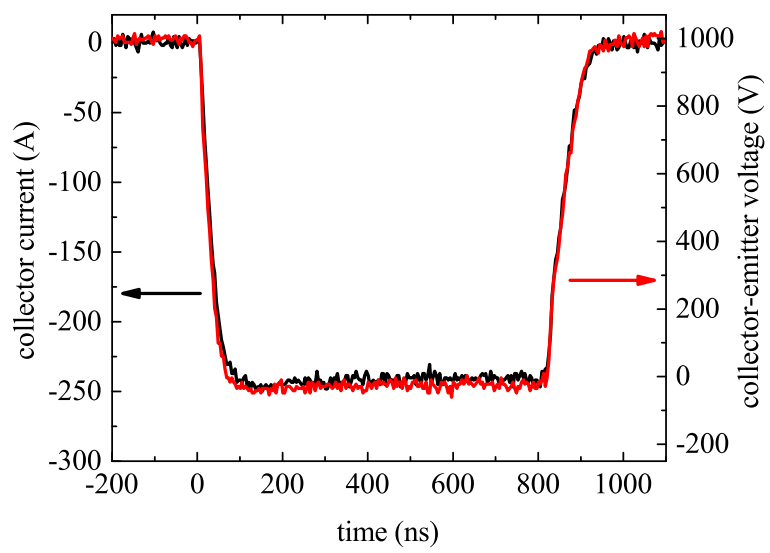

Fig. 3: The negligible phase lag between pulse current and voltage drop over the IGBT verifies the assumption of an ohmic load.

\section{RESULTS AND DISCUSSION}

\section{A. Single Pulse Experiments}

1) SiC MOSFET: The gate drive circuit has been adjusted to operate the MOSFET at a voltage across the gate capacitance between $0 \mathrm{~V}$ and $20 \mathrm{~V}$. To study the influence of the load current magnitude on the pulse shape, the device was operated at $25 \%, 50 \%$ and $80 \%$ of the maximum rated pulse current (80 A [10]). With a constant capacitor voltage of $1 \mathrm{kV}$, the load resistors were adjusted accordingly. For each current value, the gate drive voltage has been increased in steps of $20 \mathrm{~V}$ from $20 \mathrm{~V}$ to $80 \mathrm{~V}$ whereas the coupling capacitor has been adjusted to achieve $20 \mathrm{~V}$ across the gate capacitance. The measurement with $20 \mathrm{~V}$ gate drive voltage represents the standard gate drive. For this measurement, the coupling capacitor $\mathrm{C} 1$ according to fig. 1 has been shorted. In fig. 4 the resulting current measurements are shown for $80 \%$ max. rated pulse current (i.e. $64 \mathrm{~A}$ ) and varying gate drive voltage. As can be seen, the MOSFET turn-on and turn-off is nearly symmetric in all cases. The slow switching speed as achieved with the standard gate drive can be sped up to a great extend by applying an increased gate drive voltage.

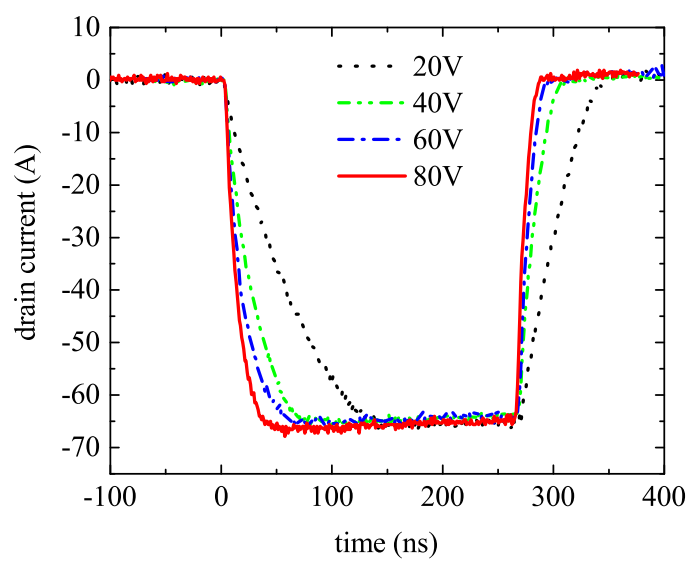

Fig. 4: Pulse shape of the drain current (C2M0080120D SiC MOSFET, $80 \%$ max. rated pulse current) in dependence of the gate drive voltage. Rise and fall time may be decreased by increasing the driver voltage.

Figure 5 shows the dependence of the calculated current rise and fall (inset) times on the gate drive voltages for different currents. The values were obtained using a $90 \%$ to $10 \%$ threshold and averaging over 200 measurements. In comparison with the datasheet values for an inductive load (20 ns rise time, $19 \mathrm{~ns}$ fall time [10]) for $20 \mathrm{~A}$ (25\% max. current) the achieved $24 \mathrm{~ns}$ rise time and $36 \mathrm{~ns}$ fall time are significantly higher. For high currents, the switching times are even slower. With increasing gate drive voltage, however, a strong decrease in switching time is observable for all curves. Using $80 \mathrm{~V}$ driving voltage, it is possible to decrease the current rise time from $71 \mathrm{~ns}$ to $20 \mathrm{~ns}$ for $80 \%$ maximum current. The accelerating effect of high driving voltages is more pronounced for high currents than it is for low currents. The achievable current rise rates for $65 \mathrm{~A}$ are increased from $0.7 \frac{\mathrm{kA}}{\mu \mathrm{s}}$ at $20 \mathrm{~V}$ to $2.6 \frac{\mathrm{kA}}{\mu \mathrm{s}}$ at $80 \mathrm{~V}$ during turn-on and from $-1.0 \frac{\mathrm{kA}}{\mu \mathrm{s}}$ at $20 \mathrm{~V}$ to $-3.5 \frac{\mathrm{kA}}{\mu \mathrm{s}}$ at $80 \mathrm{~V}$ during turn-off. Another beneficial effect of high gate drive voltages is that the rise time dependence on the current is lower, hence, the generated pulse shape has a well defined rise time regardless of the load.

2) Si IGBT: For the measurements concerning the IGBT, the gate drive circuit was adapted to achieve $17 \mathrm{~V}$ across the gate capacitance without any collector-emitter voltage applied. The resulting current pulse shapes for $250 \mathrm{~A}$ (80\% max. rated pulse current) are shown in fig. 6. Especially the current rise time is very susceptible to the gate drive voltage. The rise time is shown in fig. 7. Compared to the SiC MOSFET (fig. 5) which showed a linear decrease in current rise time with gate drive voltage, the IGBT may be sped up to a much greater 


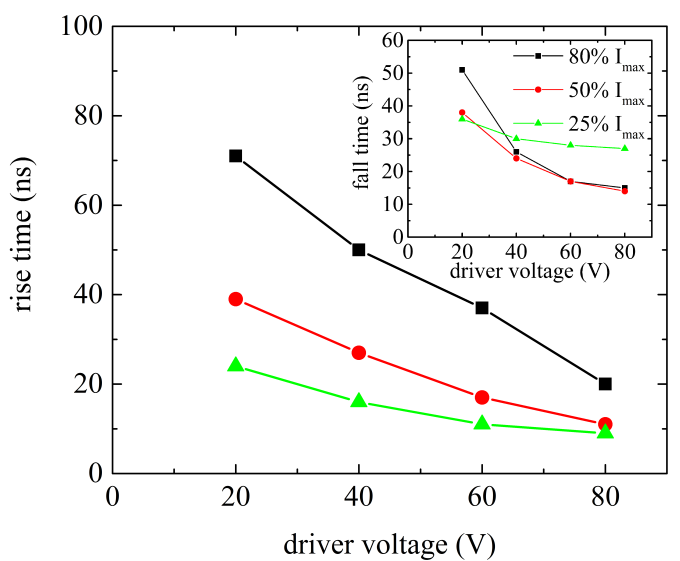

Fig. 5: Current rise times (Inset: fall times) of the SiC MOSFET C2M0080120D with varying driver voltage at $25 \%, 50 \%$ and $80 \%$ maximum rated pulse current (i.e. $20 \mathrm{~A}, 40 \mathrm{~A}$ and $64 \mathrm{~A})$. Whereas the switching times are strongly dependent on the current, increasing the gate drive voltage decreases the switching time in all cases.

extend. For $250 \mathrm{~A}(80 \%$ max. rated current) a reduction of current rise time from $400 \mathrm{~ns}$ to $49 \mathrm{~ns}$ and current fall time from $214 \mathrm{~ns}$ to $81 \mathrm{~ns}$ can be achieved using $80 \mathrm{~V}$ drive voltage. The corresponding current rise rates are increased from $0.5 \frac{\mathrm{kA}}{\mu \mathrm{s}}$ at $15 \mathrm{~V}$ to $4.2 \frac{\mathrm{kA}}{\mu \mathrm{s}}$ at $80 \mathrm{~V}$ during turn-on and from $-1.0 \frac{\mathrm{kA}}{\mu \mathrm{s}}$ at $15 \mathrm{~V}$ to $-2.6 \frac{\mathrm{kA}}{\mu \mathrm{s}}$ at $80 \mathrm{~V}$ during turn-off.

With the standard gate drive (i.e. " $15 \mathrm{~V}$ "), the switching time is strongly depended on the current to be conducted. This dependency is significantly weakened for both rise and fall time by applying a high gate drive voltage. This allows for better defined rising and falling edges and, hence, better pulse shaping capabilities of the final generator when using a variable load.

For even faster switching, connecting several devices in parallel is an option. Both devices, MOSFET and IGBT, seem suitable for parallel operation with the proposed gate drive. As a device carrying a lower current switches on faster and off slower, this negative feedback is likely to counteract current sharing imbalances between parallel devices during switching operation.

\section{B. Repetitive mode}

For high speed pulse generators, a reliable pulse shape is important. Not only pulse-to-pulse variations have to be avoided, but also device degradation over the life time of the pulse generator is an issue. Comparing the device performance over many pulses, other authors could quantify the device degradation [5]. Whereas our gate drive circuit operates within the specified values (max. $25 \mathrm{~V}$ across the gate capacitance for the MOSFET [10], $20 \mathrm{~V}$ for the IGBT [9]), the stress on the gate leads is significantly higher due to the higher gate charging current. Therefore, our test setup includes the possibility to operate the device under test for extended periods of time in repetitive mode. To cope with the increased power

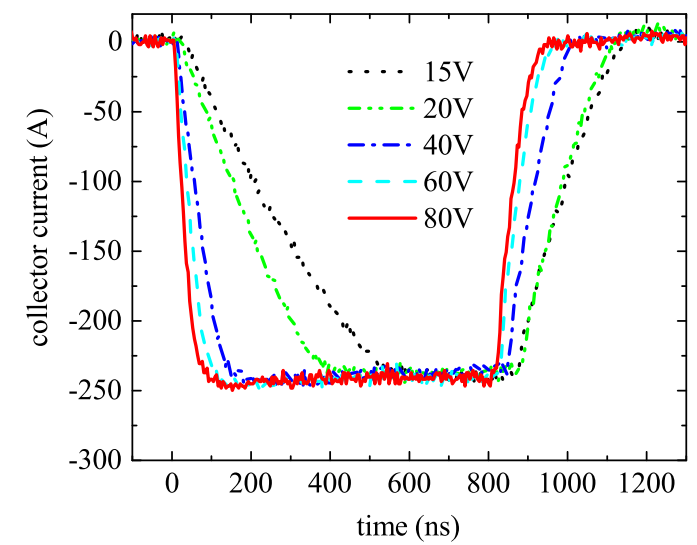

Fig. 6: Pulse shape of the collector current (NGTB40N120IHLW-D, $80 \%$ max. rated pulse current) in dependence of the gate drive voltage. Especially the current rise time can be reduced significantly by applying a high gate drive voltage.

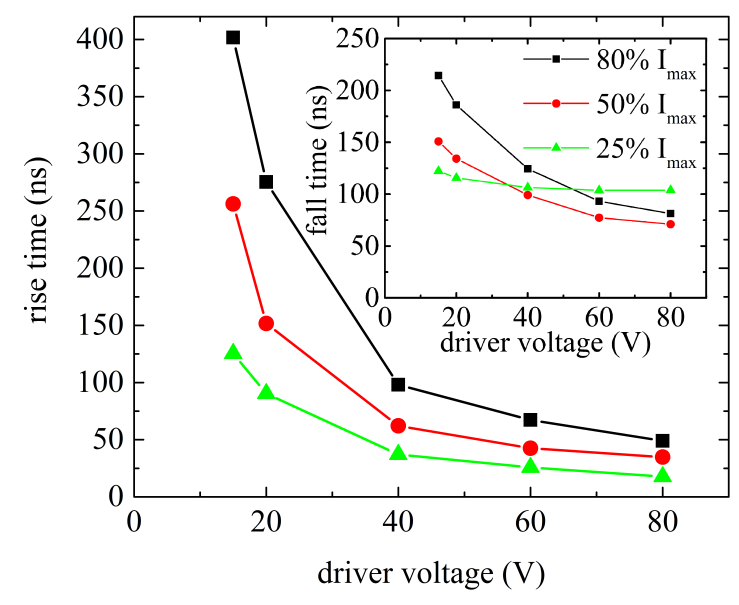

Fig. 7: Current rise times (Inset: fall times) of the IGBT NGTB40N120IHLW-D with varying driver voltage at $25 \%$, $50 \%$ and $80 \%$ maximum rated pulse current(i.e. $80 \mathrm{~A}, 160 \mathrm{~A}$ and $250 \mathrm{~A}$ ). Compared to the results for the SiC MOSFET, the increase in switching speed is much more pronounced. In addition, the variation of the current rise and fall time with the conducted current becomes smaller.

dissipation at high repetition rates, a water-cooled heatsink has been used. Its thermal resistance has been measured to be $0.18 \frac{\mathrm{K}}{\mathrm{W}}$ (case-to-water).

The measurements for the IGBT have been conducted with a pulse length of $250 \mathrm{~ns}$, a maximum current of $250 \mathrm{~A}$ at a repetition rate of $3 \mathrm{kHz}$. The gate drive voltage was set to maximum, i.e. $80 \mathrm{~V}$. In order to demonstrate the stability of the pulse shape, $10^{8}$ pulses were recorded, corresponding to $10 \mathrm{~h}$ of operation at $3 \mathrm{kHz}$.

The resulting graph is shown in fig. 8. Plotted is the current 
pulse shape at the beginning of the measurement together with the pulse shape $10 \mathrm{~h}$ later. As can be seen, both traces are identical within the resolution of the measurement. Therefore, the excellent pulse reproducibility and device stability over $10^{8}$ pulses is verified. A much longer operation time is necessary to exclude device degradation, however, the setup can now be used for long term experiments.

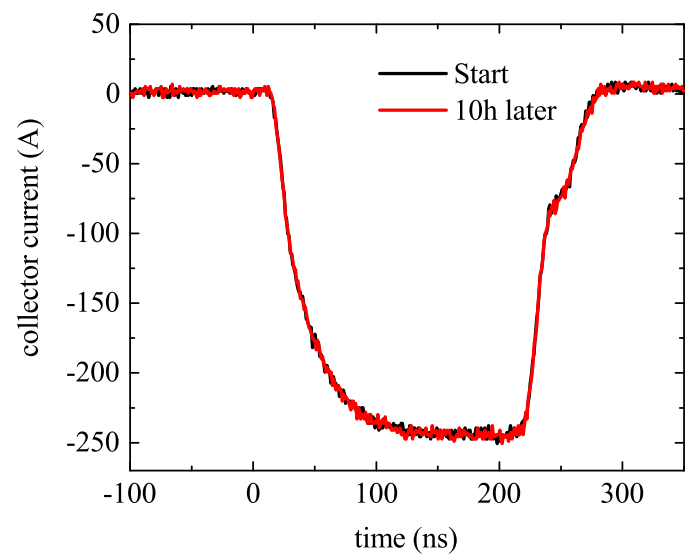

Fig. 8: Collector current pulse shape of the IGBT (NGTB40N120IHLW-D, 80\% maximum rated pulse current) before and after $10 \mathrm{~h}$ of continuous pulsing at $3 \mathrm{kHz}$ switching frequency. The identical curves show pulse shape stability over $10^{8}$ pulses.

The gate drive circuit is able to drive a device much higher frequency than the previously presented $3 \mathrm{kHz}$. Especially since many pulse generators are to perform fast pulse shaping [12], the maximum switching frequency in burst mode is important. With the IGBT under test we were able to achieve a maximum switching frequency of $3 \mathrm{MHz}(50 \%$ duty cycle) at a peak current of $250 \mathrm{~A}$ in a single device in burst mode. The resulting current trace is displayed in fig. 9.

\section{CONCLUSiON}

In this paper, our test setup for a capacitively coupled gate drive was presented. The first results have proven the flexibility of the gate drive to speed up both SiC MOSFETs as well as Si IGBTs as compared to the standard gate drive in hard switching conditions. In case of the IGBT an increase in current rise rate by a factor of 8 has been observed. The test environment thereby comprises possibilities for single pulse, burst and long term repetitive measurements. In the future, the setup will be used to benchmark new semiconductor devices for their usability in fast semiconductor-based pulse generators as well as to study the long term stability of those switching elements. Although we have not found any hints suggesting that device degradation or batch-to-batch variations influence the presented results of the tested devices, we now have the ability to identify potential problems prior to designing pulse generators.

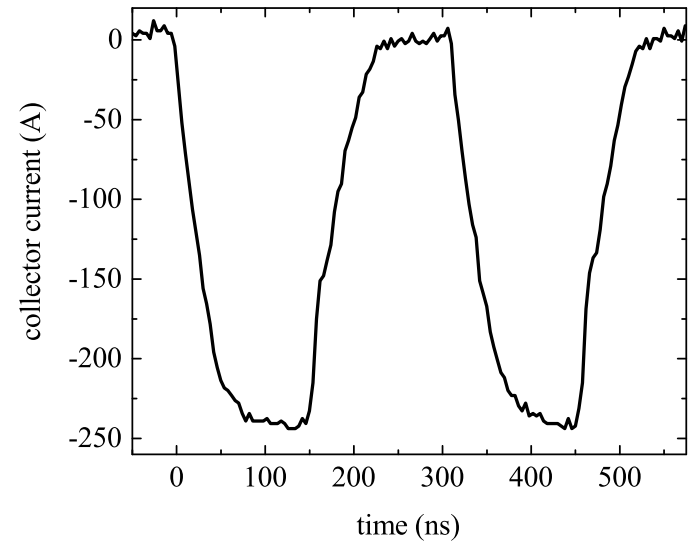

Fig. 9: Collector current of the IGBT (NGTB40N120IHLW$\mathrm{D}, 80 \%$ maximum rated pulse current) in $3 \mathrm{MHz}$ burst mode. The device under investigation is, hence, suitable for reliable, fast repetitive switching when employing the proposed gate drive circuit.

\section{REFERENCES}

[1] M. Sack, S. Keipert, M. Hochberg, M. Greule, and G. Mueller, "Design considerations for a fast stacked-mosfet switch," IEEE Transactions on Plasma Science, vol. 41, no. 10, pp. 2630-2636, 2013.

[2] A. Volke and M. Hornkamp, IGBT modules: Technologies, driver and application. Munich: Infineon Technologies AG, 2011.

[3] N. Telzhensky and I. Zeltser, Fast transition high voltage modulator using SiC MOSFETs connected in parallel. Presented at: 28th Convention of Electrical and Electronics Engineers in Israel (IEEEI), 2014, Eilat, Israel, 2014. [Online]. Available: http://ieeexplore.ieee.org/iel7/6996094/7005728/07005844.pdf? arnumber $=70058404$, Accessed: Nov. 15, 2015

[4] W. Jiang, K. Yatsui, K. Takayama, M. Akemoto, E. Nakamura, N. Shimizu, A. Tokuchi, S. Rukin, V. Tarasenko, and A. Panchenko, "Compact solid-state switched pulsed power and its applications," Proceedings of the IEEE, vol. 92, no. 7, pp. 1180-1196, 2004.

[5] J. A. Schrock, W. B. Ray, K. Lawson, A. Bilbao, S. B. Bayne, S. L. Holt, L. Cheng, J. W. Palmour, and C. Scozzie, "High-mobility stable 1200-v, 150-a 4h-sic dmosfet long-term reliability analysis under high current density transient conditions," Power Electronics, IEEE Transactions on, vol. 30, no. 6, pp. 2891-2895, 2015

[6] R. Bayerer and D. Domes, "Parasitic inductance in gate drive circuits," PCIM Europe Conference Proceedings, pp. 473-477, 2012.

[7] M. N. Nguyen, R. L. Cassel, J. E. deLamare, and G. C. Pappas, "Gate drive for high speed, high power igbts," PPPS-2001: Pulsed Power Plasma Science 2001, Vols I and II, Digest of Technical Papers, 2001.

[8] Fairchild Semiconductor, "Datasheet fdt86102lz mosfet." [Online]. Available: www.fairchildsemi.com/datasheets/FD/FDT86102LZ.pdf, Accessed: Nov. 15, 2015

[9] ON Semiconductor, "Datasheet for igbt ngtb40n120ihlwg." [Online]. Available: http://www.onsemi.com/pub/Collateral/NGTB40N120IHLWD.PDF, Accessed: Nov. 15, 2015

[10] Cree Inc., "Datasheet for sic mosfet c2m0080120d." [Online]. Available: http://www.wolfspeed.com///media/Files/Cree/Power/Data Sheets/C2M0080120D.pdf, Accessed: Nov. 15, 2015

[11] M. Sack and G. Müller, EMC aspects in the layout of a fast semiconductor switch. Presented at: Electromagnetic Compatibility in Automotive Engineering, GMM Specialist Conference 2014; Dusseldorf; Germany; 11 March 2014 through 13 March 2014.

[12] W. Jiang, H. Sugiyama, and A. Tokuchi, "Pulsed power generation by solid-state ltd," IEEE Transactions on Plasma Science, vol. 42, no. 11, pp. 3603-3608, 2014. 


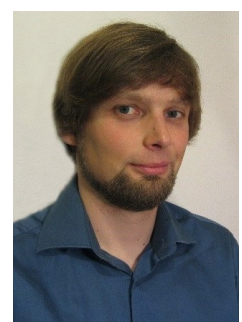

Martin Hochberg received the B.Sc. degree in Physics from Frankfurt University, Germany in 2011 and the Master 2 Recherche in Physics for Energetics from Grenoble INP, France together with the M.Sc. Degree in Physics from Karlsruhe Institute of Technology, Germany in 2014.

Currently, he is with Karlsruhe Institute of Technology, Institute for Pulsed Power and Microwave Technology, pursuing his $\mathrm{PhD}$. His research interests include fast semiconductor switches, the design of pulsed power generators and the generation of

intense electron beams.

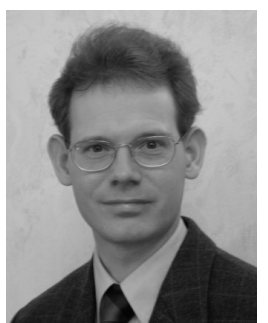

Martin Sack Martin Sack received the Diploma and Ph.D. degree in electrical engineering from Karlsruhe University, Karlsruhe, Germany, in 1995 and 2002, respectively. Since 2002 he is with Karlsruhe Institute of Technology, Institute for Pulsed Power and Microwave Technology (IHM) and since 2006 he is head of the research group "Pulsed Power Technologies and Systems" at IHM. His current research interests cover pulse generators and circuits for pulsed power applications, including industrial applications, fast switching, pulsed electric field (PEF) treatment of plant cells, measurement technology related to pulsed power and PEF treatment, and electromagnetic compatibility. Martin Sack gives a lecture on "Electronic Devices and Electromagnetic Compatibility" at KIT, Department for Electrical Engineering and Information Technology.

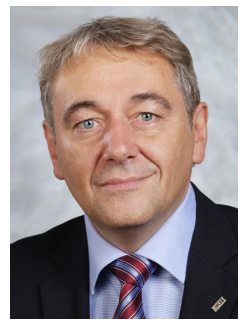

Georg Mueller received the Diploma degree in physics and $\mathrm{Ph}$. D. degree from the University of Karlsruhe, Germany, in 1990 and 1999 respectively. Since 1990, he was with the Research Centre Karlsruhe, where he worked in different research fields including thin film deposition by channel spark, plasma and electron beam diagnostics, development of multi point explosive emission cathodes, transport of large area powerful pulsed electron beams and surface modification by pulsed electron beams (GESA-process). He is author and co-author of more than 200 publications in peer reviewed journals, conference proceedings and two book chapters. He is member of the European Pulse Power Society, the International Advisory Committees of the HLMC-, BEAMS- and EAPPCconferences and member of the contact expert group of the European Commission on transmutation and the OECD/NEA expert group on HLM. Since 2006 he is Deputy Director and Head of the Pulsed Power Department of the Institute for Pulse Power and Microwave Technology at Karlsruhe Institute of Technology (KIT). He is responsible and is involved in research and development of pulsed power applications in the field of electrodynamic fragmentation of solid dielectric materials, material surface modification by pulsed electron beams, electroporation of biological cell membranes by pulsed electric fields and basic research in bioelectrics. 